\title{
Supercritical Fluid Extraction in the Undergraduate Laboratory
}

\author{
Rebecca K, Toghiani, Hossein Toghiani, and Jay Jones' \\ Department of Chemical Engineering \\ Mississippi State University \\ P.O. Box 9595 \\ Mississippi State, MS 39762
}

\section{Introduction}

Thermodynamics experiments are often an overlooked component in the chemical engineering undergraduate laboratory curriculum. This is in part due to the expense of necessary equipment and also due to the length of time and degree of precision required to perform experiments to demonstrate textbook principles. At Missippi State University, a major portion of the renovation effort over the past six years has been the development and incorporation of experiments focusing on emerging technologies such as supercritical fluid extraction, students can best be exposed to these technologies in a laboratory setting. Supercritical fluid extraction requires the use of phase equilibrium principles and thus, experiments focusing on this technology provide exposure both to its use as an emerging separations technology and to the underlying thermodynamic relationships.

Supercritical fluid extraction technology has been developing for the past fifteen years and while commercial applications in the U.S. are few and limited in scope, opportunities are ever expanding. In recent years, use of this technology in the environmental restoration sector, the food and drug sector, as well as the chemical industry have been discussed in the literature [Johnston, 1989; Taylor, 1995]. Supercritical fluid extraction does not give rise to secondary waste streams in environmental applications. This is in contrast to incineration or leaching, where monitoring and secondary disposal costs may contribute significantly to the overall process cost. The use of supercritical fluids in the food and drug industry arises from the need to separate thermally labile materials from complex mixtures. The use of supercritical carbon dioxide, in particular, allows the necessary separation to be conducted at much lower temperatures than would be realized in other separation processes.

The opportunities for laboratory experiments are diverse in nature and differ in their degree of difficulty and implementation. Solute volubility in supercritical solvents is crucial to the success of this technology in an industrial application, The measurement of volubility for various solutes, typically low volatility solids and liquids, in supercritical fluids has been reported in the literature [McHugh and Paulaitis, 1980; Chang and Morrell, 1984; Kurnik et al,, 1981]. An understanding of the various factors which affect this volubility can be gained by the undergraduate student through properly designed experiments.

\section{Background and Theory}

Supercritical fluid extraction exploits the unique behavior displayed by a material at a temperature and pressure slightly above its critical point. At a temperature slightly above the critical point of the solvent, the properties of the fluid vary smoothly from liquid-like behavior to gas-like behavior without the abrupt change in

\footnotetext{
${ }^{1}$ Present Address: PPG Industries, Inc., PO, Box 1000, Lake Charles, LA 70602
} 
properties associated with a phase transition below the critical temperature [Williams, 1981]. This smooth variation allows the density of the supercritical fluid, and thus its capacity for solutes, to be varied by changing the pressure. Desirable effects are also observed in the transport properties. Difusivities are enhanced compared to liquid phase diffusivities while viscosity decreases markedly compared to the liquid phase. Solubilities are enhanced making the supercritical fluid behave similar to a liquid solvent in its capacity for solutes. These effects combine to give supercritical fluids unique properties with respect to their ability to extract solutes from complex matrices [Hawthorne and Miller, 1987].

The basis for predicting the volubility of a solute in a supercritical fluid solvent is the equivalence of fugacities for the particular solute in each phase:

$\hat{f}_{1}^{\mathrm{s}}=\hat{f}_{\mathrm{i}}^{\mathrm{f}}$

where the superscript $s$ represents the solid phase and $f$ the supercritical fluid phase. If the volubility of the supercritical fluid in the solid phase is assumed negligible, then the fugacity of the solute in the solid phase, $\hat{f}_{i}^{s}$, is equal to the fugacity of the pure solute, $f_{i}^{s}$. The fugacity of the pure solute in the solute phase is evaluated using [Modell and Reid, 1983]:

$f_{\mathrm{i}}^{\mathrm{s}}=\mathrm{P}_{\mathrm{i}}^{\mathrm{sat}}\left(\frac{f_{\mathrm{i}}^{\mathrm{sat}}}{\mathrm{P}}\right) \exp \left[\int_{\mathrm{P}_{\mathrm{i}}^{\text {sat }}}^{\mathrm{P}}\left(\frac{\mathrm{V}_{\mathrm{i}}^{\mathrm{s}}}{\mathrm{RT}}\right) \mathrm{dP}\right]$

The molar volume of the pure solid, $\mathrm{V}, \mathrm{s}$, is assumed constant. The fugacity of the pure solute in the saturated state, $f_{i}^{\text {sat }}$, is combined with the pressure in the denominator to form the fugacity coefficient of the pure solute in the saturated state, $\phi_{i}^{\text {sat }}$. Integration of equation (2) with these substitutions yields [Modell and Reid, 1983]:

$f_{i}^{\mathrm{s}}=\mathrm{P}_{\mathrm{i}}^{\mathrm{sat}} \phi_{\mathrm{i}}^{\mathrm{sat}} \exp \left[\frac{\mathrm{V}_{\mathrm{i}}^{\mathrm{s}}}{\mathrm{RT}}\left(\mathrm{P}-\mathrm{P}_{\mathrm{i}}^{\mathrm{sat}}\right)\right]$

For many of the low volatility compounds of interest in environmental applications and in particular, for naphthalene, the fugacity coefficient for the pure solute in the saturated state is very nearly unity due to its extremely low vapor pressure, a feature possessed by many of these compounds at ambient conditions. For naphthalene, the vapor pressure at $35^{\circ} \mathrm{C}$ is 0.00279 bar,

The fugacity of the solute in the supercritical fluid phase is given by:

$\hat{f}_{\mathrm{i}}^{\mathrm{f}}=\mathrm{y}_{\mathrm{i}} \hat{\phi}_{\mathrm{i}} \mathrm{P}$

where $y_{i}$ is the mole fraction of solute in the supercritical fluid phase, also defined as the volubility of the solute in the supercritical fluid; $\hat{\phi}_{i}$ is the fugacity coefficient for the solute in the supercritical fluid phase; and $P$ is the system pressure.

Combination of equations (3) and (4) yields an expression for the volubility of the solute, $y_{i}$, in the supercritical fluid [Modell and Reid, 1983]: 
$\mathrm{y}_{\mathrm{i}}=\left(\frac{\mathrm{P}_{\mathrm{i}}^{\mathrm{sat}}}{\mathrm{P}}\right)\left(\frac{1}{\hat{\phi}_{\mathrm{i}}}\right) \exp \left[\frac{\mathrm{V}_{\mathrm{i}}^{\mathrm{s}}\left(\mathrm{P}-\mathrm{P}_{\mathrm{i}}^{\mathrm{sat}}\right)}{\mathrm{RT}}\right]$

The ideal volubility of the solute, a function of temperature and pressure, is represented by the first bracketed term in the expression. For many solutes of interest, the ideal volubility will be extremely low due to the low vapor pressure of the solute and the high pressure required to achieve the supercritical state for the solvent. Non-ideal behavior of the supercritical fluid phase is represented by the second bracketed term in the expression. The last term is the Poynting factor which represents the effect of pressure on the solid phase. The last two bracketed terms, when combined, are known as the enhancement factor. This factor represents the increase in volubility due to the solvent's supercritical state. Enhancement factors are often quite large; with values ranging from $10^{4}$ to $10^{6}$ being cited in the literature [Brennecke, 1989].

The fugacity of the solute in the supercritical fluid can be evaluated using a cubic equation of state such as the Redlich-Kwong-Soave or the Peng Robinson equations. In this paper, the Peng-Robinson equation is utilized. The Peng-Robinson equation for a mixture is [Modell and Reid, 1983]:

$P=\frac{R T}{V-b_{M}}-\frac{a_{M}}{V\left(V+b_{M}\right)+b_{M}\left(V-b_{M}\right)}$

where $\mathrm{V}$ is the molar volume of the mixture while $a_{M}$ and $b_{M}$ are mixture dependent parameters. For the PengRobinson equation of state, these mixture parameters are evaluated from mixing rules where the pure component analogues, calculated from the critical properties, the acentric factor, and the reduced temperature for each species, are combined through appropriate combinatorial relationships. The standard Peng-Robinson mixing rules are utilized in this work.

$a_{M}=\sum_{i=1}^{N} \sum_{j=1}^{N} y_{i} y_{j} a_{i j}$

$\mathrm{b}_{\mathrm{M}}=\sum_{\mathrm{i}=1}^{\mathrm{N}} \mathrm{y}_{\mathrm{i}} \mathrm{b}_{\mathrm{i}}$

with: $\left.a_{i j} \neq 1-\delta_{i j}\right) \sqrt{a_{i} a_{j}}$ and $a_{i i}=a_{1}$. The binary interaction parameter, $\delta_{i j}$, is specific for the $i-j$ binary pair. In these expressions, the $\mathrm{a}_{\mathrm{i}}$ and $\mathrm{b}_{\mathrm{i}}$ represent the pure component parameter values which are evaluated using:

$\mathrm{a}_{\mathrm{i}}\left(\omega, \mathrm{T}_{\mathrm{r}}\right)=\mathrm{a}\left(\mathrm{T}_{\mathrm{c}}\right) \alpha\left(\omega, \mathrm{T}_{\mathrm{r}}\right)$

$\mathrm{a}\left(\mathrm{T}_{\mathrm{c}}\right)=\frac{0.45724 \mathrm{R}^{2} \mathrm{~T}_{\mathrm{c}}^{2}}{\mathrm{P}_{\mathrm{c}}}$

$\alpha\left(\omega, T_{r}\right)=\left[1+\left(0.37464+1.54226 \omega-0.26992 \omega^{2}\right)\left(1-\sqrt{T_{r}}\right)\right]^{2}=\left[1+\kappa\left(1-\sqrt{T_{r}}\right)\right]^{2}$

$\mathrm{b}_{\mathrm{i}}=\frac{0.07780 \mathrm{RT}_{\mathrm{c}}}{\mathrm{P}_{\mathrm{c}}}$

The Peng-Robinson equation is often written in terms of the compressibility, $\mathrm{Z}(=\mathrm{PV} / \mathrm{RT})$ : 
where $A$ and $B$ are defined as:

$$
\begin{aligned}
& A=\frac{a_{M} P}{(R T)^{2}} \\
& B=\frac{b_{M} P}{R T}
\end{aligned}
$$

The use of this equation of state for evaluation of the fugacity coefficient of the solute in a supercritical fluid is well-documented in the literature [McHugh and Paulaitis, 1980; Brennecke, 1989]. Following the notation of Modell and Reid [1983], the fugacity coefficient for species i in solution is found through:

$$
\ln \left(\hat{\phi}_{i}\right)=\frac{b_{i}}{b_{M}}(Z-1)-\ln (Z-B)+\frac{A}{2 \sqrt{2} B}\left(\frac{2 \sum_{k} y_{k} a_{i k}}{a_{M}}-\frac{b_{i}}{b_{M}}\right) \ln \left[\frac{Z+B(1-\sqrt{2})}{Z+B(1+\sqrt{2})}\right]
$$

The prediction of volubility by this set of equations requires an iterative approach, one well-suited for adaptation in a spreadsheet program such as Quattro-Pro or Excel. In general, the temperature and pressure are known, as well as an estimate of the volubility, $\mathrm{y}_{\mathrm{i}}$. If experimental data is not available for use as the initial estimate, then an estimate can be computed by assuming that ideal behavior is valid ( $\mathrm{i}, \mathrm{e}, \mathrm{y},=\mathrm{P}_{\mathrm{i}}^{\text {sat }} / \mathrm{P}$ ). The estimated $\mathrm{y}$, can then be used with the given temperature to evaluate the pure component and mixture parameters. The molar volume of the mixture is computed using the given $\mathrm{T}$ and $\mathrm{P}$ with the mixture parameters through solution of the cubic equation of state, equation (6). The compressibility can then be calculated and substituted into the expression for the fugacity coefficient, equation (16), along with the necessary pure component and mixture parameters. An alternate route is to evaluate the compressibility directly from equation (13) instead of the volume from equation (6). The predicted volubility can then be calculated through equation (5). The iterative procedure is continued until the predicted volubility at the end of an iteration is equal to the estimate at the start of the iteration to within some prescribed tolerance.

\section{Experimental Apparatus and Procedure}

Volubility experiments at MSU are carried out using a Dense Gas Management (DGM) System and a 300 $\mathrm{cm}^{3}$ extraction vessel, both designed and constructed by Marc Sims SEE, Inc. (Berkeley, CA). This system is designed to safely withstand pressures of up to 6000 psig and temperatures of up to $100^{\circ} \mathrm{C}$. Thus, a variety of supercritical solvents could be handled with the equipment. A schematic of the experimental apparatus is shown in Figure 1. Major components of the apparatus include a feed line filter and chiller, a liquid metering pump, a heated extraction vessel, a heated expansion valve, a separation chamber and solvent flow measurement devices.

The DGM system allows controlled delivery of solvent with independent control of pressure and of flowrate. The equipment is compatible with a variety of supercritical solvents. Typically, experiments are carried out with carbon dioxide as the solvent because it is inexpensive, it easily disengages from the solute upon expansion to ambient conditions and the supercritical state for carbon dioxide can be achieved at moderate pressure and relatively low temperature (73. 76 bar, $304,2 \mathrm{~K})$, 
Liquid carbon dioxide is supplied to the system from a standard gas cylinder equipped with a dip tube. The carbon dioxide is cooled to below the saturation temperature at the supply pressure by passing it through a chiller supplied with $2 \mathrm{LPM}$ of cooling water at $\mathrm{O}^{\circ} \mathrm{C}$. The cooled carbon dioxide then passes to the LDC Analytical miniPump (Model 2396 Duplex) where it is pumped to the desired operating pressure. A regulator adjusts the flow of carbon dioxide through the recycle loop allowing delivery of the solvent at pressures of up to $6000 \mathrm{psig}$. The pump eflluent passes through $1 / 16^{\prime \prime}$ diameter high pressure tubing and enters the bottom of the extraction vessel.

The tubular extraction vessel, $300 \mathrm{~cm}^{3}$ in volume, is equipped with a cartridge system to allow use of samples of smaller volumes $\left(25,50\right.$ and $\left.100 \mathrm{~cm}^{3}\right)$. The cartridges are cylindrical and equipped with frits at both ends which allow the solvent to pass through the sample, yet prevent entrainment of solid solute, When the cartridge system is in use, glass beads are placed in the lower section of the extractor and serve to heat the solvent to the desired operating temperature as well as to distribute the solvent throughout the extraction vessel to prevent channeling. A heating mantle surrounding the tubular extraction allows the regulation of the extraction temperature. The mantle is controlled through an Omega temperature indicator/controller housed in the DGM console, A type $\mathrm{J}$ thermocouple coupled to an Omega Model 115 JC digital indicator registers the temperature of the solvent immediately prior to its passage through the sample.

The supercritical solvent passes through the sample and the solute/solvent mixture then leaves the extraction vessel and passes through a heated expansion valve. It is essential that the supercritical carbon dioxide pass through the cartridge containing the solid naphthalene at a sufficiently low flowrate to ensure equilibration between the naphthalene and carbon dioxide. This required flowrate was determined through a series of experiments where the solvent flowrate was varied and the experimental volubility calculated. When the experimental volubility no longer varied with the flowrate, the flowrate was deemed sufficiently low to ensure ample time for equilibration between the solute and the solvent, The equilibrated naphthalene/carbon dioxide mixture then passes into a pre-weighed separator where the naphthalene precipitates. The solvent carbon dioxide expands to atmosphere pressure and passes through a flow totalizer and a flowmeter before venting.

\section{Results and Discussion}

In the students' experiments, the volubility of naphthalene, well documented in the literature [Tsekhanskaya et al., 1964; McHugh and Paulaitis, 1980; Chang and Morrell, 1984], is measured at two temperatures and two pressures. Experimental conditions are chosen to match existing literature data. The volubility is determined from the experimental measurements. The weight of naphthalene, $m_{\text {solutc }}$, collected during a volubility run was determined from the pre- and post-experimental weights of the separation chamber. To ensure that no naphthalene was entrained in the exiting carbon dioxide, the outlet of the separator was filled with glass wool to trap any naphthalene which did not settle, Rarely was this a problem due to the low flowrate of carbon dioxide passing through the separator. The volume of solvent, $\mathrm{V}_{\mathrm{TOT}}$, corresponding to the collected solute was determined by a flow totalizer. Multiplication by the molar density of carbon dioxide, $\widetilde{\rho}_{\mathrm{CO}_{2}}$,yields the moles of solvent corresponding to the collected solute, The experimental volubility is then given by:

$\mathrm{y}_{1}=\frac{\mathrm{m}_{\text {solute }} / \mathrm{MW}_{\text {solute }}}{\mathrm{m}_{\text {solute }} / \mathrm{MW}_{\text {solute }}+\mathrm{V}_{\mathrm{TOT}} \widetilde{\mathrm{\rho}}_{\mathrm{CO}_{2}}}$

The experimentally determined solubilities are then compared to literature data, A comparison of data collected in the laboratory with literature data is shown in Figures 2 and 3. The reproduction of literature data in the 
laboratory, while time-consuming, provides the student with an appreciation for the patience and attention to detail that one must demonstrate to obtain data of this type.

As part of the laboratory experiment/report, the students must also develop a spreadsheet or computer program which calculates the volubility for naphthalene in supercritical carbon dioxide. A typical Excel spreadsheet is shown in Figure 4. This spreadsheet was developed by an undergraduate student to perform the complex and lengthy calculations involved in the evaluation of predicted volubility. Input for the spreadsheet include solute and solvent properties, the experimental temperature and pressure and an estimate of the volubility, $\mathrm{y}_{1}$. Using these input data, the Peng-Robinson mixture parameters are calculated. The compressibility of the mixture, Z, corresponding to the experimental temperature, pressure and the estimate of the volubility, is determined through use of the SOLVER function in Excel, This implementation makes use of the iterative capabilities of Excel, by setting the estimated volubility contained in cell B 13 equal to the value calculated through use of the Peng-Robinson equation (equation 5, cell B29), This circular reference is resolved through iteration until the estimated value is equal to the predicted value to within a user-specified tolerance. The development of this spreadsheet, while time-intensive, is useful in that predicted solubilities for a variety of conditions can be easily and routinely calculated. The inclusion of cells where the pertinent critical and physical property values for the solute and solvent alike are entered result in a very versatile spreadsheet that can be used to predict the volubility of different solutes in supercritical carbon dioxide or in a variety of supercritical solvents.

\section{Conclusion}

Through this combination of experiment and calculation, the student becomes familiar with supercritical fluid extraction and the relative importance of temperature and pressure on the volubility of a low volatility material in a supercritical solvent. The development of a spreadsheet capable of carrying out the complex iterative calculations required for the prediction of volubility in the supercritical fluid allows the student to fully exploit the power of these useful applications, The opportunity to examine this phenomena in the laboratory setting provides the student with a visual and valuable experience which combines the area of novel separations technology with the relevant thermodynamic principles.

\section{References}

Brennecke, J. F., Ph.D. Dissertation, University of Illinois - Champaign-Urbana, 1989,

Chang, H., Morrell, D. G., J. Chem. Eng. Data, 30, 74, (1984).

Hawthorne, S. B., Miller, D, J., Anal. Chem., 59, 1705, (1987).

Johnston, K. P., 'New Directions in Supercritical Fluid Science and Technology', in Supercritical Fluid Science and Technology, Johnston, K. P., Penninger, J, M, L., editors, (American Chemical Society, Washington, D. C.: 1989).

Kurnik, R. T., Holla, S. J., Reid, R. C., J. Chem. Eng. Data, 26,47, (1981),

McHugh, M., Paulaitis, M. E., J. Chem. Eng. Data, 25, 326, (1980).

Modell, M, Reid, R. C., Thermodynamics and Its Applications, (2nd edition, Prentice Hall Publishing,

Englewood Cliffs, NJ: 1983),

Sandier, S.I., Chemical and Engineering Thermodynamics, (2nd edition, John Wiley Publishing Co, New York, NY: 1989),

Taylor, L. T., Introduction to Supercritical Fluid Extraction, (R\&D Magazine, Des Plaines, IL: 1995).

Tsekhanskaya, Y. V., Iomtev, M. B., Mushkina, E. V., Russ. J. Phys. Chem., $\underline{38}(9)$, 1173, (1964).

Williams, D. F., Chem. Eng. Sci., 36(1 1), 1796, (1981). 
REBECCA K. TOGHIANI is an Assistant Professor at MSU. She received her B, S. Ch.E., M. S. Ch.E. and Ph.D. degrees in Chemical Engineering from the University of Missouri-Columbia. She is a member of ASEE, AIChE, WEPAN, ISA, AAUW, and an honorary member of the Golden Key National Honor Society. Her research interests include thermodynamics of liquid mixtures and novel separation technology.

HOSSEIN TOGHIANI is an Associate Professor at MSU. He received his B. S. Ch.E., M. S. Ch.E. and Ph.D. degrees in Chemical Engineering from the University of Missouri-Columbia. A member of AIChE, ASEE and ISA, he was selected as 1994-95 Professor of the Year by departmental seniors at MSU. His research interests include analysis of plasma torch gaseous effluents and surface modification of rubber derived from scrap tires.

JAY JONES, MSU's Dow Outstanding Junior for 1994, received his B. S. Ch.E, from MSU in May 1995. Currently employed as a Technical Assistant in Silica Products at PPG Industries, Inc., in Lake Charles, LA, he also worked for Dow Chemical in Plaquemine, LA and First Chemical Corp in Jackson, MS while pursuing his degree. He is a member of AIChE, TAPPI, Golden Key, Omega Chi Epsilon, Phi Kappa Phi, and Tau Beta Pi. 


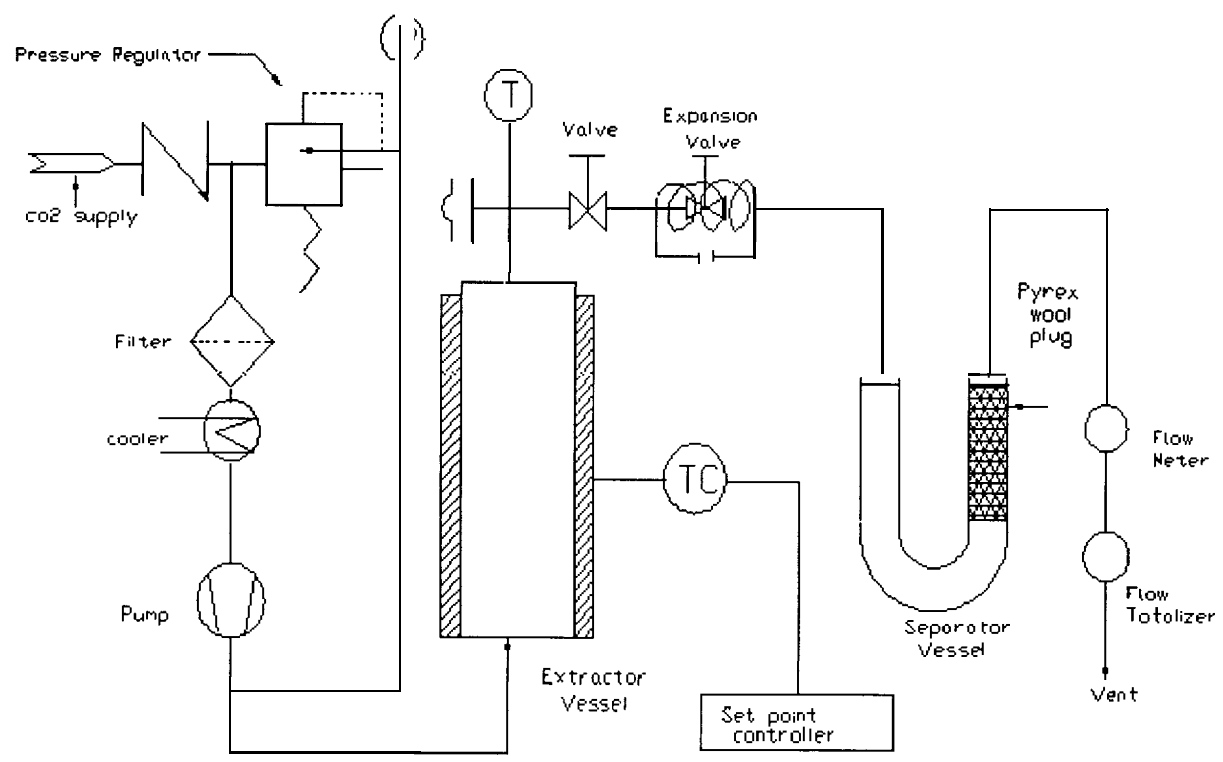

Figure 1, Schematic Diagram of Supercritical Fluid Extraction Apparatus

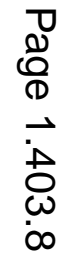




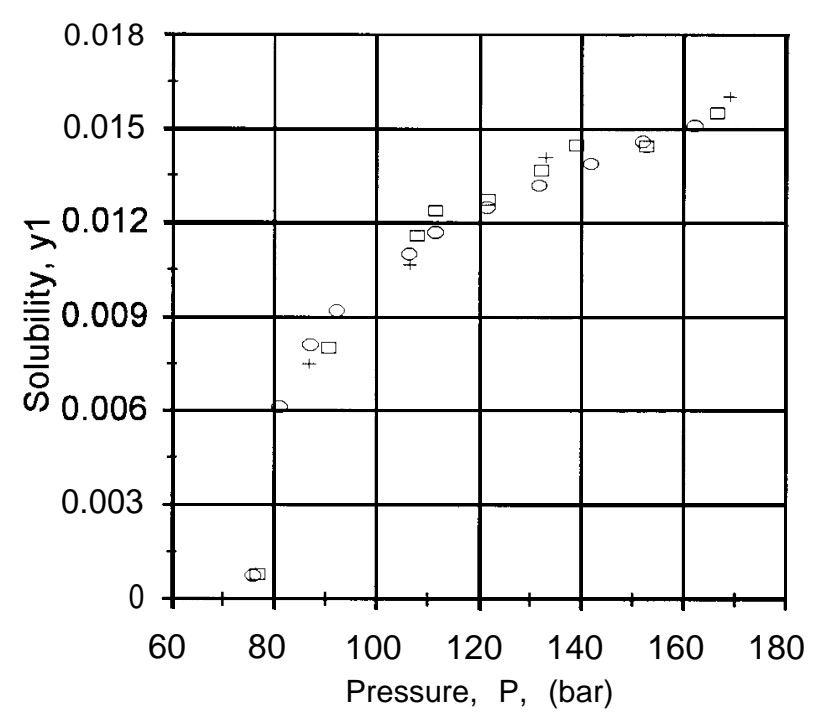

Figure 2, Volubility of Naphthalene in Supercritical Carbon Dioxide. Comparison of Experimental Data with Literature Data at $35^{\circ} \mathrm{C}$. 0 - Experimental; •l Tsekhanskaya et al., (1964); - McHugh and Paulaitis, (1980).

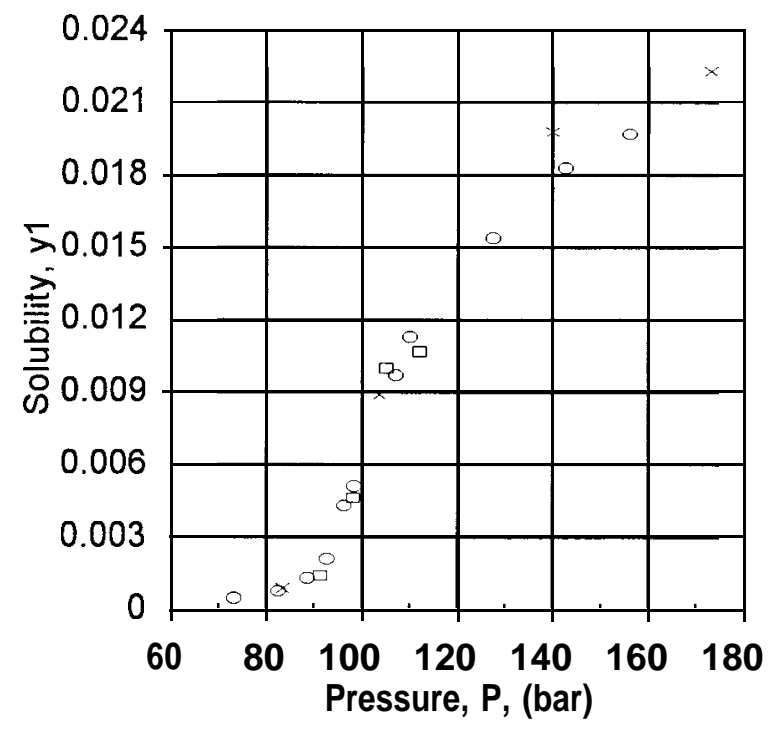

Figure 3. Volubility of Naphthalene in Supercritical Carbon Dioxide. Comparison of Experimental Data with Literature Data at $45^{\circ} \mathrm{C}$. O - Experimental; • 1 Tsekhanskaya et al., (1964); x - Chang and Morrell, (1984). 


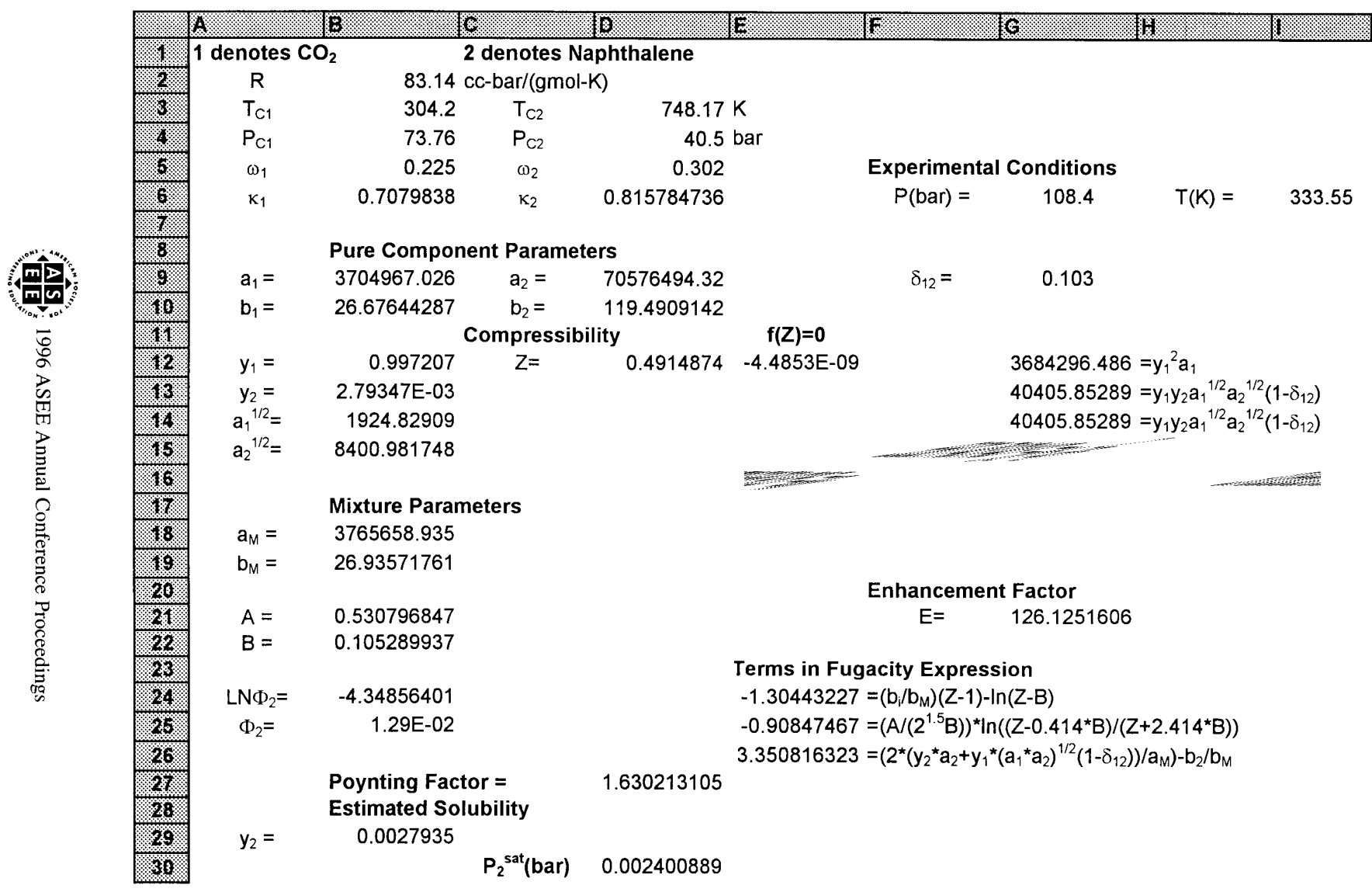

Figure 4. Excel Spreadsheet Developed for Prediction of Volubility in Supercritical Fluid 